\title{
Magnetic resonance imaging evaluation of incidentally detected hyperechoic liver lesions: comparison of two modalities in terms of detection, diagnosis, and morphological features
}

\author{
Gokhan Soker $^{1 *}$ (D), Serife Leblebisatan ${ }^{1}$, Okan Dilek ${ }^{1}$ (D), Huseyin Akkaya ${ }^{1}(\mathbb{D}$, \\ Ibrahim Inan² (1), Omer Kaya ${ }^{3}$, Cengiz Yilmaz ${ }^{1}$ (D), Bozkurt Gulek ${ }^{1}$ (1)
}

\section{SUMMARY}

OBJECTIVE: This study aimed to investigate and compare the ultrasonography and contrast-enhanced magnetic resonance imaging characteristics of incidentally detected hyperechoic focal liver lesions.

METHODS: Seventy-four patients (29 males and 45 females) who had undergone a B-mode ultrasonography and contrast-enhanced magnetic resonance imaging examination were included in this study. A total of 91 hyperechoic lesions detected on ultrasonography were evaluated. The ultrasonography features of these hyperechoic lesions were recorded, and the results were compared with those acquired from contrast-enhanced magnetic resonance imaging. The results were compared statistically using the Shapiro-Wilk, McNemar, and Wilcoxon signed-rank tests.

RESULTS: A corresponding lesion was found on contrast-enhanced magnetic resonance imaging in 72 of the 91 (79.1\%) hyperechoic lesions detected on ultrasonography. Forty-one (56.9\%) of the magnetic resonance imaging-defined lesions were typical hemangiomas, while 10 (13.9\%) were focal steatosis areas and 4 (5.6\%) were diagnosed with hepatocellular carcinoma. In contrast, 6 lesions (8.3\%) were diagnosed as simple hepatic cysts, 4 (5.6\%) as sclerosing hemangioma, 2 (2.8\%) as thrombosed hemangioma, 1 (1.4\%) as focal nodular hyperplasia, 1 (1.4\%) as hamartoma, 2 (2.8\%) as hydatid cysts, and 1 (1.4\%) as hepatic lipoma. No statistically significant differences were found between ultrasonography and magnetic resonance imaging in terms of the segmental classification of the true positive lesions based on contour structures and lesion area measurements ( $p=0.558, p=0.375$, and $p=0.636$, respectively).

CONCLUSIONS: Incidentally detected hyperechoic zones may not necessarily be detected on magnetic resonance imaging. This may be secondary to focal hepatic steatosis or false interpretation of the radiologist. Lesions requiring therapy must be considered in the differential diagnosis.

KEYWORDS: Ultrasound imaging. Incidental finding. Hemangioma. Liver steatosis. Magnetic resonance imaging.

\section{INTRODUCTION}

Advancements in technical ultrasonography (US) standards and the increasing number of abdominal US examinations performed globally have also led to an increase in the number of incidentally detected hyperechoic liver lesions on US. These lesions sometimes create confusion in the differential diagnosis and necessitate further imaging examinations, such as magnetic resonance imaging (MRI). US echogenicity increases

\footnotetext{
'University of Health Sciences, Adana Teaching and Research Hospital, Department of Radiology - Adana, Turkey.

${ }^{2}$ Centermed Advanced Imaging Center - Istanbul, Turkey.

${ }^{3}$ Cukurova University, Faculty of Medicine, Department of Radiology - Adana, Turkey.

*Corresponding author: gsoker@hotmail.com

Conflicts of interest: the authors declare there is no conflicts of interest. Funding: none.

Received on September 19, 2021. Accepted on September 20, 2021.
} 
in many instances, including the presence of steatosis, calcification, fibrosis, and gas ${ }^{1}$. Benign lesions, such as hemangioma, focal steatosis, lipoma, hamartoma, and focal fibrosis, as well as malignant lesions, e.g., primary and secondary malignancies, are considered in the differential diagnosis of hyperechoic liver lesions ${ }^{2,3}$. This study presents the results of the dynamic MRI examinations of focal hyperechoic liver lesions detected incidentally on US.

\section{METHODS}

\section{Patient selection and evaluation}

This retrospective study was approved by our institutional ethical committee and was carried out in accordance with the principles of the Declaration of Helsinki and the Good Clinical Practice guidelines. Notably, 74 patients who had undergone a B-mode US and dynamic MRI examination of the liver were included in this study. These patients who had undergone a US examination for various reasons were incidentally detected to have hyperechoic lesions. Patients with known liver tumors, with extrahepatic malignancies with the potential to metastasize to the liver, with a chronic liver disease such as cirrhosis, and with an interval of 60 days or longer between the US and MRI examinations were excluded from this study. The MRI examinations of the patients whose focal hyperechoic liver lesions were incidentally detected on US were evaluated by two expert radiologists with 16 and 14 years of experience in their profession. The results were obtained based on consensus. Both radiologists evaluated the dynamic MRI images to define the characteristics of the lesions and diagnose them in light of clinical data and laboratory test results. The radiologists who evaluated the MRI studies had no information about the size, location, and other characteristic properties of the lesions detected on US.

\section{Sonographic evaluations}

The US examinations of all patients were performed using a Philips Epiq 7G device (Andover, MA, USA) with an abdominal transducer. No sonographic contrast material was utilized. The patients underwent their US examinations following a fasting period of $6 \mathrm{~h}$. The US examinations were performed during deep inspiration, with the patients holding their breath. Certain features of hyperechoic liver lesions detected on US, such as the largest diameter, segmental location, contour properties, and compression characteristics of adjacent structures, were documented. In patients with more than one lesion, the data of each lesion were recorded separately according to its segmental location.

\section{Magnetic resonance imaging evaluations} All MRI examinations were undertaken with a 1.5 Tesla scanner (Ingenia; Philips Healthcare, The Netherlands). The following sequences were obtained for all patients: axial and coronal balanced turbo field echo T2-weighted $(\mathrm{T} 2 \mathrm{~W})$, axial spectral attenuated inversion recovery $\mathrm{T} 2 \mathrm{~W}$, diffusion-weighted imaging, ADC mapping, and dual-echo fast field echo (FFE). In addition, the T1-weighted (T1W) fat-suppressed gradient echo thrive sequence was utilized in all patients both with and without intravenous gadolinium administration $(0.1 \mathrm{mmoL} / \mathrm{kg}$ body weight). Following the gadolinium injection, contrast-enhanced images were acquired at the arterial, portal, and venous phases. The acquisition parameters of the dual-echo sequence were as follows: field of view (FOV), $450 \times 398 \mathrm{~mm}$; matrix size, $280 \times 248$; slice thickness, $5 \mathrm{~mm}$; flip angle, $75^{\circ}$; repetition time (TR), 106 $\mathrm{ms}$; in-phase echo time (TE), $4.6 \mathrm{~ms}$; and out-of-phase TE, $2.3 \mathrm{~ms}$. The acquisition parameters of the dynamic MRI sequence were selected as follows: FOV, $450 \times 401 \mathrm{~mm}$; matrix size, $300 \times 229$; slice thickness, $6 \mathrm{~mm}$; flip angle, $10^{\circ}$; TR, $4.2 \mathrm{~ms}$; and TE, $2.1 \mathrm{~ms}$.

An ovoid or geographical lesion located adjacent to the gallbladder fossa, falciform ligament, or capsule with no mass effect, appearing as hyperintense in the in-phase image and hypointense in the out-of-phase image on the dual-echo FFE sequence, was defined as an area of focal fatty infiltration. Focal lesions with the following MRI characteristics were defined as hemangiomas: hypointense in the T1W images, hyperintense in the $\mathrm{T} 2 \mathrm{~W}$ images, peripheral nodular contrast enhancement in the arterial dynamic phase, and centripetal contrast enhancement in the portal and late venous phases. Hyperintensity in the T2W sequence, peripheral nodular contrast enhancement starting in the arterial phase, and hypointense areas in the portal and late phases were interpreted to indicate thrombosed hemangiomas. T2W hyperintensity with mild peripheral nodular contrast enhancement in the late phase and capsular contraction was defined as a sclerosing hemangioma. Lesions demonstrating $\mathrm{T} 1 \mathrm{~W}$ hypointensity, T2W mild hyperintensity, hyperintensity in the dynamic arterial phase, and iso-intensity or mild hyperintensity in the portal and late venous phases were defined as focal nodular hyperplasia. T1W hypointense and T2W substantially hyperintense lesions showing no contrast enhancement at the dynamic phases were defined as simple hepatic cysts. T1W hypointense lesions showing substantial hyperintensity in the $\mathrm{T} 2 \mathrm{~W}$ sequence and thin peripheral contrast enhancement in the dynamic series were diagnosed as hamartomatous cysts. Lesions appearing as hypointense in T1W and hyperintense in $\mathrm{T} 2 \mathrm{~W}$ sequences and possessing a peripheral hypointense rim 
that demonstrated contrast enhancement in the late phase and had curvilinear internal structures were interpreted as hydatid cysts. Lesions that appeared as hypointense foci in T1W and moderately hyperintense foci in $\mathrm{T} 2 \mathrm{~W}$ sequences and demonstrated hyperenhancement in the dynamic arterial phase and a quick washout following the arterial phase were diagnosed as hepatocellular carcinomas (HCCs). Lesions demonstrating hyperintensity in both $\mathrm{T} 1 \mathrm{~W}$ and $\mathrm{T} 2 \mathrm{~W}$ sequences, hypointensity in fat-saturated T2 sequences, and a hypointense band of India ink artifact in the out-of-phase sequence were considered as hepatic lipomas.

The dimensions, contour characteristics, and segmental localization of the lesions were documented from the MRI images. All the lesions were evaluated using the MRI sequence where they were most apparent. Contrast-enhanced dynamic imaging was performed for hemangiomas and HCCs, whereas T2W sequences were obtained for simple and hydatid cysts. Multiple measurements were obtained, and the longest diameter was noted. In patients with more than one lesion, the results of each lesion were recorded separately. Lesions that could not be categorized according to the MRI characteristics were excluded from this study.

\section{Statistical analysis}

SPSS version 21.0 was used for the statistical analysis of the data. Categorical variables were recorded as numbers and percentages. The Shapiro-Wilk test was utilized for the spatial evaluation of numeric parameters. Variables showing a normal distribution were defined with the mean and standard deviation parameters, while those not demonstrating a normal distribution were expressed as median, minimum, and maximum values. The McNemar test was conducted for the comparison of categorical data obtained from MRI and US, whereas the Wilcoxon signed-rank test was performed for the comparison of nonparametric numerical data.

\section{RESULTS}

Twenty-nine (39.2\%) of 74 patients included in this study were males and $45(60.8 \%)$ were females. The mean age of the patients was $45.58 \pm 14.06$ years. The interval time between the US and MRI examinations of the patients ranged from 0-55 days, with a median value of three days. On US, a total of 103 lesions were detected, of which 12 could not be categorized on MRI, and thus they were excluded from this study. The US and MRI findings of the 91 lesions detected on US were compared.

For $72(79.1 \%)$ of the 91 hyperechoic lesions detected on US, a corresponding lesion was detected on MRI.
No corresponding lesion was identified on MRI in the remaining 19 lesions. No statistically significant difference was present between the segmental localizations of the sonographic and MRI positive lesions that were defined on both US and MRI ( $\mathrm{p}=0.558)$. Only five (5.49\%) of the 91 lesions were the segments of localizations differed according to the diagnostic modality (US or MRI).

Forty-one (56.9\%) of the MRI-defined lesions were typical hemangiomas, whereas 10 (13.9\%) were focal steatosis areas. Four (5.6\%) lesions were diagnosed as HCCs. The diagnostic categorization of the MRI-defined lesions is demonstrated in Table 1.

Sixty-seven (73.6\%) of the US-diagnosed 91 lesions were defined as regularly contoured and $24(26.4 \%)$ as irregularly contoured. The MRI numbers corresponding to these US numbers were $53(73.6 \%)$ and $19(26.4 \%)$, respectively, in the 72 lesions detected at MRI. No statistically significant difference was found between US and MRI in terms of the classification of the lesions according to their contour characteristics $(\mathrm{p}=0.375)$.

Area calculations were performed by the proper multiplication of the long and short axes of the lesions. The results of these lesion measurements were recorded as minimum 37.5 $\mathrm{mm}^{2}$, maximum $4.928 \mathrm{~mm}^{2}$, and median $251 \mathrm{~mm}^{2}$ for US, and minimum $42 \mathrm{~mm}^{2}$, maximum $4420 \mathrm{~mm}^{2}$, and median $226.5 \mathrm{~mm}^{2}$ for MRI. There was no statistically significant difference between the US and MRI results in terms of lesion area measurements $(\mathrm{p}=0.636)$.

\section{DISCUSSION}

The incidental finding of a focal hyperechoic lesion on US is a frequently encountered situation, and a proper differential

Table 1. Diagnosis of the lesions according to MRI findings.

\begin{tabular}{l|c|c} 
Diagnosis & Number & $(\%)$ \\
\hline Typical hemangioma & 41 & 56.9 \\
\hline Sclerosing hemangioma & 4 & 5.6 \\
\hline Thrombosed hemangioma & 2 & 2.8 \\
\hline Focal nodular hyperplasia & 1 & 1.4 \\
\hline Focal steatosis & 10 & 13.9 \\
\hline Hamartoma & 1 & 1.4 \\
\hline Hepatocellular carcinoma & 4 & 5.6 \\
\hline Simple hepatic cyst & 6 & 8.3 \\
\hline Hydatid cyst & 2 & 2.8 \\
\hline Hepatic lipoma & 1 & 1.4 \\
\hline Total & 72 & 100 \\
\hline
\end{tabular}

MRI: magnetic resonance imaging. 
diagnosis is not always possible by US alone. The literature indicates that these focal hyperechoic lesions mostly represent a hemangioma ${ }^{4}$. However, the differential diagnosis list also includes various other lesions, most of which are benign. Although US and MRI have different advantages and disadvantages in detecting lesions, MRI plays a problem-solving role in these situations.

In recent studies, it has been shown that MRI is superior to the detection of focal liver lesions compared with US and computed tomography due to its high soft tissue resolution ${ }^{5}$. To the best of our knowledge, there is no study in the English language literature assessing the sensitivity of MRI in the detection of incidentally detected hyperechoic lesions. In this study, MRI was not able to detect 19 of the 91 focal hyperechoic lesions (20.8\%) observed during US examinations. This may be due to the interpretation error of the radiologist who performed the US examination. Another explanation may be that these lesions might be visible during US examinations due to the slight sonographic contrast created by the focal lesion over the liver parenchyma, but their MRI signals might not be strong enough to create MRI contrast sufficient for visualization (Figure 1). In addition, focal fatty infiltration may be associated with increased hepatic iron accumulation and can hamper the detection of focal fatty infiltration ${ }^{5}$.

The gold standard for the detection of liver steatosis is a biopsy ${ }^{6}$, but due to its invasive nature, hepatic steatosis is usually screened by US to perform a qualitative evaluation $^{7}$. In contrast, qualitative and quantitative evaluations are undertaken with the utilization of certain MRI sequences ${ }^{8}$. It has been reported that MRI has $76.7-90 \%$ sensitivity and $87.1-91 \%$ specificity in the imaging diagnosis of hepatic steatosis $^{9-12}$. Opposed-phase imaging performed within the dual-echo sequence in MRI can make the proper diagnosis of hepatic steatosis by demonstrating the signal loss, and techniques such as MRI spectroscopy and the newly developed proton density fat fraction can evaluate liver fat quantitatively ${ }^{8,13}$. Focal hepatic steatosis is usually detected on US as a hyperechoic focus and may be mistaken for a metastasis and vice versa ${ }^{14,15}$. The focal fatty infiltrations of the liver are described as geographically bordered lesions without a compression effect, usually localized in the gallbladder fossa, segment 4 , and the areas neighboring the falciform ligament and portal vein ${ }^{14}$. Similarly, in this study, 9 of the $10(90 \%)$ lesions defined as focal steatosis were located at segment 4 . Seven $(70 \%)$ lesions had geographical contours, while none had a compression effect. These results show that even though the diagnostic spectrum of hyperechoic focal liver lesions detected on US comprises many pathologies involving malignancies, MRI may be unnecessary in the presence of lesions with typical contour characteristics, which do not possess a hypoechoic halo or have a compression effect on the adjacent structures. Although it is not yet fully understood and moderately differentiated, HCCs may

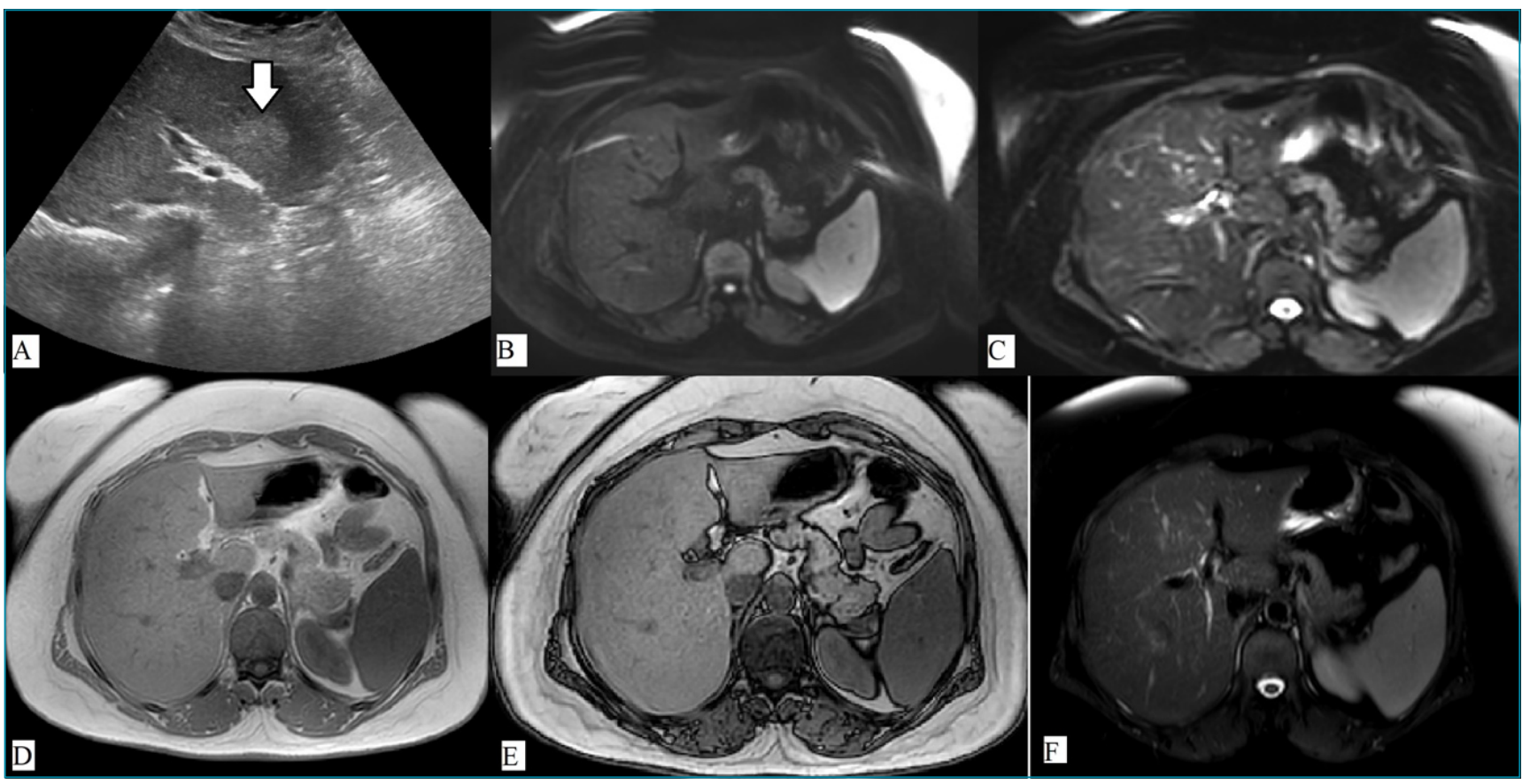

Figure 1. (A) Ultrasonography clearly demonstrates a hyperechoic area in the left lobe of the liver. (B), (C), (D), (E), and (F) No lesion is detected on diffusion weighted imaging, dual echo, and T2-weighted images. Images of a 43-year-old woman. 
include fat and appear hyperechoic on US5. The literature indicates that small HCC lesions may have a higher chance of appearing hyperechoic on US ${ }^{16}$. In this study, a total of four lesions in two patients had received a diagnosis of HCC. Three of these lesions were $2 \mathrm{~cm}$ or less in size, and all comprised focal heterogeneous areas (Figure 2).

The number of studies in the literature concerning focal liver lesions detected incidentally on US is rather limited. In a retrospective study performed by Kaltenbach et al., the incidence of hepatic benign lesions detected by re-scanning the previously performed US examinations was found to be $15.1 \%$. In that study, the most frequent focal pathology was reported to be focal fatty sparing, with an incidence of $6.3 \%{ }^{17}$. According to the results of this study, the most frequent hyperechoic lesions were typical hemangiomas (56.9\%), followed by the lesions of focal hepatic steatosis (13.9\%). We should emphasize that among the diagnoses acquired by MRI were benign pathologies such as hydatid cysts and lipomas, as well as malignant pathologies such as HCC. This is an important finding necessitating a thorough investigation of the history and clinical findings of patients in addition to their US imaging findings (Figure 3).

In this study, area measurements, together with their contour characteristics and segmental localizations, were compared between the US and MRI examinations. A carefully performed US examination can provide very similar results to an MRI examination. In contrast, it is also clear that US is usually sufficient in the follow-up of certain characteristics of previously diagnosed lesions, such as their numbers and lesion area measurements. To the best of our knowledge, in the English language literature, these lesion characteristics have not been previously compared these two imaging modalities. Our findings should be verified by similar further studies performed with larger patient cohorts.

The relatively low number of patients included in this study is a limitation. We think that further studies that will be performed with larger patient groups will disclose the presence of other benign and malignant lesions. Another limitation of this study is that the diagnoses were made based on MRI findings, and biopsy procedures could not be performed due to ethical reasons. The evaluation of follow-up imaging findings may overcome this problem.

\section{CONCLUSIONS}

Incidentally detected hyperechoic zones may not necessarily be detected on MRI secondary to mild focal hepatic steatosis or false interpretation of the radiologist. Although hemangioma is the usual suspect in the focal hyperechoic lesions of the liver detected on US, lesions requiring therapy must also be considered in the differential diagnosis. A thorough evaluation of these patients can be undertaken by acquiring detailed clinical data, scrutinizing sonographic and MRI findings, and performing a biopsy procedure if necessary.

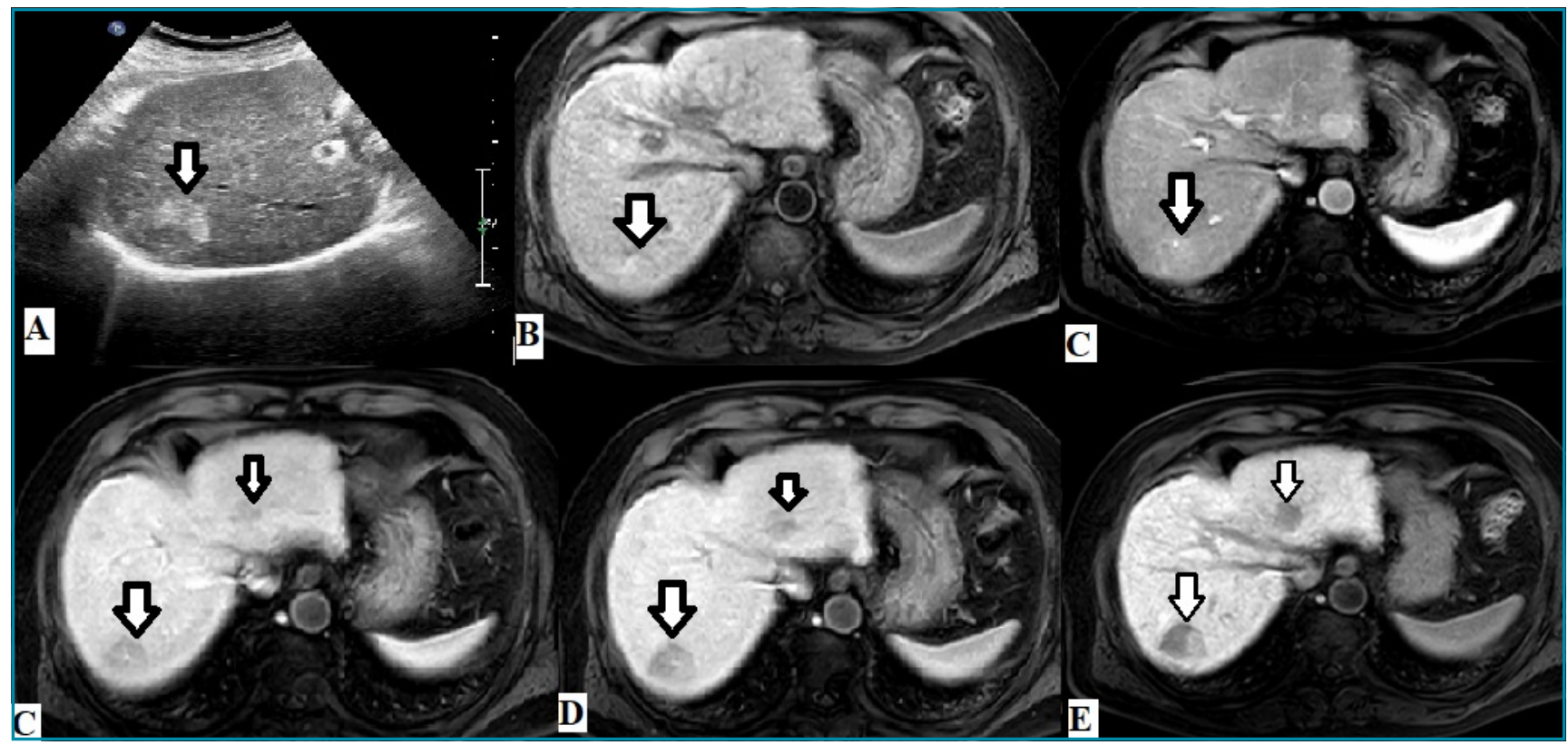

Figure 2. (A) A hyperechoic lesion with hypoechoic areas at segment 7 on ultrasonography (another hyperechoic lesion is not shown). (B) The lesion is slightly hyperintense on the axial T1-weighted image. (C), (D), and (E) Axial T1-weighted image shows arterial hyperenhancement and wash out in portal and venous phases. (E) Hepatobiliary phase lesions are hypointense. Images of a 70-year-old man with hepatocellular carcinoma. 


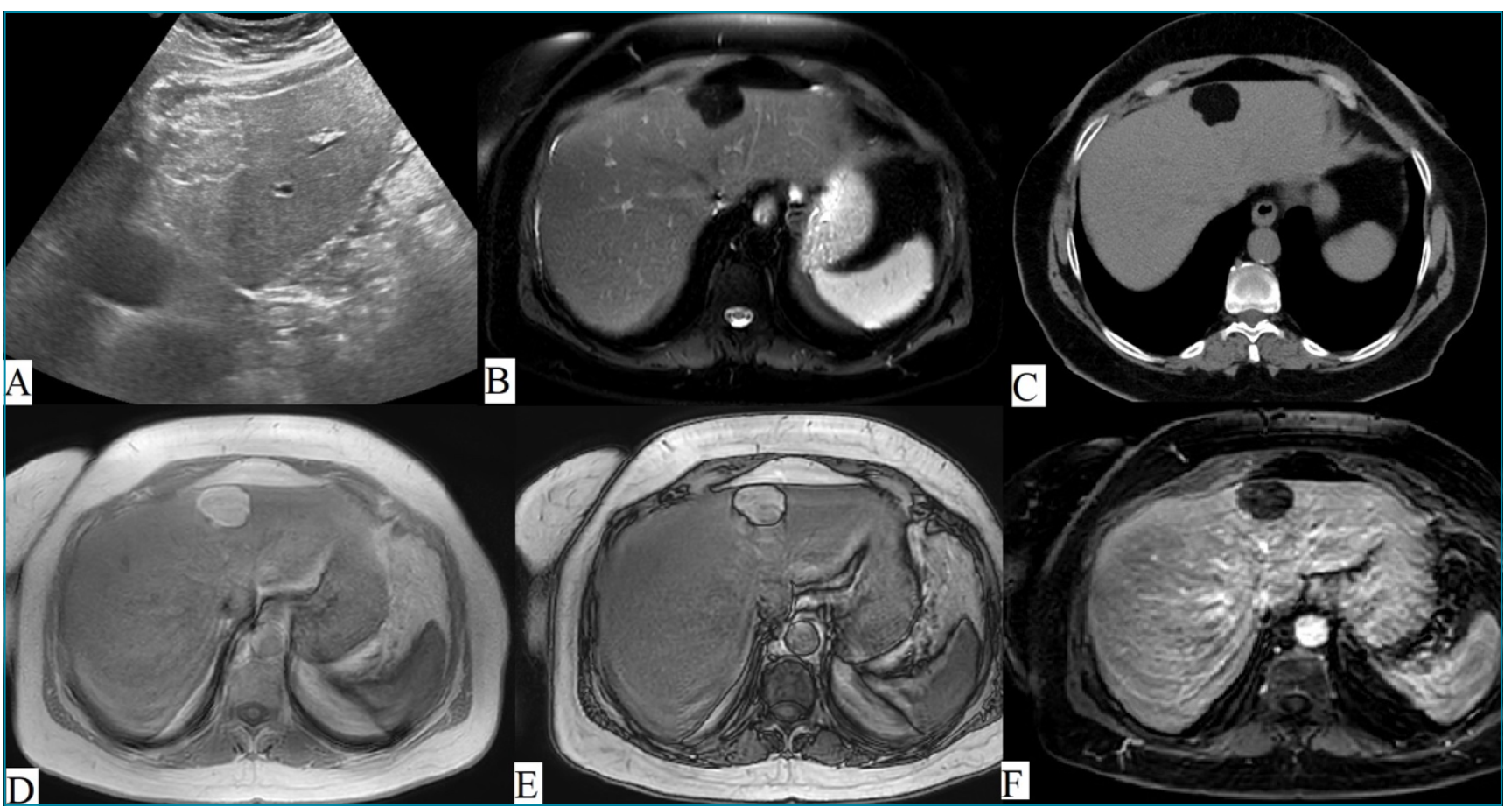

Figure 3. (A) Ultrasonography image reveals a hyperechoic lesion at segment 2 of the liver. (B) Axial fat-saturated T2-weighted image shows a hypointense lesion. (C) In the axial computed tomography image, the lesion is hypodense. (D) and (E) Dual gradient echo in-phase and opposed-phase images demonstrate a hyperintense lesion with a chemical shift artifact at the periphery of the lesion. (F) There is no contrast enhancement on the axial T1-weighted image. Images of a 58-year-old woman with a hepatic lipoma.

\section{AUTHORS' CONTRIBUTIONS}

GS: Conceptualization, Data curation, Writing - original draft. SL: Conceptualization, Data curation. OD: Conceptualization,
Data curation. HA: Data curation. OK: Data curation. II: Conceptualization, Formal Analysis. CY: Supervision. BG: Writing - review \& editing.

\section{REFERENCES}

1. Kim Y, Jung $C$, Jeong WK, Kim YS, Koh BH, Cho OK, et al. Hyperechoic hepatic nodules: correlation of findings from sonography, CT, and pathologic analysis. J Clin Ultrasound. 2004;32(8):399-410. https://doi.org/10.1002/jcu.20057

2. Rocha CATD, Kamada LMK, Andrade Filho PH, Villaverde IA, Shiro JYB, Silva Junior JMD. Ultrasonographic evaluation of gastric content and volume: a systematic review. Rev Assoc Med Bras (1992). 2020;66(12):1725-30. https://doi. org/10.1590/1806-9282.66.12.1725

3. Saraci G, Truta A. Hyperechogenic liver lesion. Maedica (Bucur). 2011;6(3):213-4. PMID: 22368700

4. Rocha SMS, Yamanari MGI, Matsuoka MW, Almeida GC, Sakamoto FA, Suzuki L. Focal hyperechoic liver lesions in children: far beyond hemangiomas - pictorial essay. Radiol Bras. 2020;53(2):122-8. https://doi.org/10.1590/01003984.2018.0119

5. Costa AF, Thipphavong S, Arnason T, Stueck AE, Clarke SE. Fat-containing liver lesions on imaging: detection and differential diagnosis. AJR Am J Roentgenol. 2018;210(1):68-77. https:// doi.org/10.2214/AJR.17.18136
6. Tüten MF, Özdamarlar U, Yılmabaşar MG, Kutlu Y, Hayırlıoğlu DA, Kuru LI. Nonalkolik karaciğer yağlanmasında histopatolojik bulguların MR ile korelasyonu. Medeni Med J. 2014;29(2):82-7. https://doi.org/10.5222/J. GOZTEPETRH.2014.082

7. Kramer H, Pickhardt PJ, Kliewer MA, Hernando D, Chen GH, Zagzebski JA, et al. Accuracy of liver fat quantification with advanced ct, mri, and ultrasound techniques: prospective comparison with MR spectroscopy. AJR Am J Roentgenol. 2017;208(1):92-100. https://doi.org/10.2214/AJR.16.16565

8. Ingraham C, Bhargava P, Otjen J, Medverd JR, Vaidya S Multiple echogenic liver masses from multifocal nodular steatosis in a 55-year-old male with chronic hepatitis C. Radiol Case Rep. 2015;5(3):399. https://doi.org/10.2484/ rcr.v5i3.399

9. Shannon A, Alkhouri N, Carter-Kent C, Monti L, Devito R, Lopez $R$, et al. Ultrasonographic quantitative estimation of hepatic steatosis in children with NAFLD. J Pediatr Gastroenterol Nutr. 2011;53(2):190-5. https://doi.org/10.1097/ MPG.0b013e31821b4b61 
10. Lee SS, Park SH, Kim HJ, Kim SY, Kim MY, Kim DY, et al. Non-invasive assessment of hepatic steatosis: prospective comparison of the accuracy of imaging examinations. J Hepatol. 2010;52(4):579-85. https://doi.org/10.1016/j.jhep.2010.01.008

11. Werven JR, Marsman HA, Nederveen AJ, Smits NJ, Kate FJ, Gulik $\mathrm{TM}$, et al. Assessment of hepatic steatosis in patients undergoing liver resection: comparison of US, CT, T1-weighted dual-echo MR imaging, and point-resolved $1 \mathrm{H}$ MR spectroscopy. Radiology. 2010;256(1):159-68. https://doi.org/10.1148/radiol.10091790

12. Pilleul F, Chave G, Dumortier J, Scoazec JY, Valette PJ. Fatty infiltration of the liver. Detection and grading using dual T1 gradient echo sequences on clinical MR system. Gastroenterol Clin Biol. 2005;29(11):1143-7. https://doi.org/10.1016/s0399-8320(05)82179-7

13. Zhang YN, Fowler KJ, Hamilton G, Cui JY, Sy EZ, Balanay $M$, et al. Liver fat imaging-a clinical overview of ultrasound, CT, and MR imaging. Br J Radiol. 2018;91(1089):20170959. https://doi.org/10.1259/bjr.20170959
14. Metin NO, Karaosmanoğlu AD, Metin $Y$, Karçaaltıncaba $M$. Focal hypersteatosis: a pseudolesion in patients with liver steatosis. Diagn Interv Radiol. 2019;25(1):14-20. https://doi. org/10.5152/dir.2018.17519

15. Karcaaltincaba M, Akhan O. Imaging of hepatic steatosis and fatty sparing. Eur J Radiol. 2007;61(1):33-43. https:// doi.org/10.1016/j.ejrad.2006.11.005

16. Rapaccini GL, Pompili M, Caturelli E, Covino M, Lippi ME, Beccaria S, et al. Hepatocellular carcinomas $<2 \mathrm{~cm}$ in diameter complicating cirrhosis: ultrasound and clinical features in 153 consecutive patients. Liver Int. 2004;24(2):124-30. https://doi. org/10.1111/j.1478-3231.2004.0903.x

17. Kaltenbach TE, Engler P, Kratzer W, Oeztuerk S, Seufferlein T, Haenle MM, et al. Prevalence of benign focal liver lesions: ultrasound investigation of 45,319 hospital patients. Abdom Radiol (NY). 2016;41(1):25-32. https://doi.org/10.1007/ s00261-015-0605-7 